1985-12

\title{
On the structure of wave fronts in nonlinear dissipative media
}

\section{Barenblatt, GI}

http://hdl.handle.net/10026.1/9746

10.1007/bf00250915

Archive for Rational Mechanics and Analysis

Springer Science and Business Media LLC

All content in PEARL is protected by copyright law. Author manuscripts are made available in accordance with publisher policies. Please cite only the published version using the details provided on the item record or document. In the absence of an open licence (e.g. Creative Commons), permissions for further reuse of content should be sought from the publisher or author. 
Offprint from "Archive for Rational Mechanics and Analysis",

Volume 87, Number 4, 1985, pp. 293-303

(C) Springer-Verlag 1985

Printed in Germany

\title{
On the Structure of Wave Fronts in Nonlinear Dissipative Media
}

\author{
G. I. Barenblatt, M. Ya. Ivanov, \& G. I. Shapiro \\ Dedicated to Jerald Ericksen
}

Wave fronts are well known to be the regions of space where the properties of motion vary sharply in a certain direction. This means that one of the components of the gradients of density, velocity, stress, etc., at the wave fronts exceeds essentially not only other components but also corresponding gradients outside the fronts. The large gradients require that the analysis of motion within the fronts (the front structure) be based on equations more detailed than those sufficient in the surroundings. Meanwhile these more detailed equations admit simplification of the boundary-layer type because the fronts are necessarily slender in one direction. In the "external" approximation the fronts are represented by discontinuity surfaces (Figure 1, a).

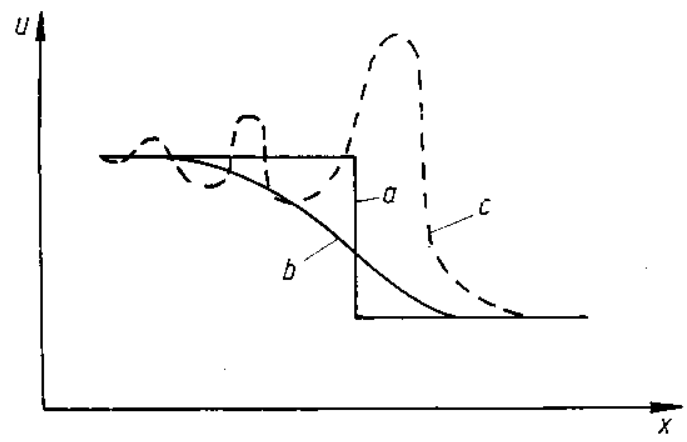

Fig. 1. (a) In external approximation the front is a discontinuity surface. (b) In the dispersionless media the front has the form of a smoothed step. (c) The dispersion leads to oscillations of the characteristics of the motion

A complete formulation of the theory of gasdynamic fronts was presented in the classical paper of N. E. Kochin (1926). Gasdynamic fronts show no effects of dispersion; the nonlinear effects in these fronts are balanced by viscosity and 
other dissipative effects only. Therefore these fronts have a monotonic structure of smoothed step type (Figure 1, b). In more complicated media, dispersion can influence the structure of the wave fronts to a great extent.

In particular, such a situation is characteristic of fronts in the ocean as well as in other stratified aquifiers. Oscillation of properties of the motion across the front is typical there (Figure $1, \mathrm{c}$ ). Such behaviour was found by R. Z. SAGDEEV (1964) for collisionless shock fronts in plasma and by A. V. GAPONOV, L. A. OstrovSKY \& G. I. FreIDMAN (1967) for electromagnetic shock waves in solids etc.

Nonlinear waves in dispersive media have been considered by many authors. Reviews of basic results as well as references may be found in KARPMAN (1973), Whitham (1974), Leibovich \& SeEbass (eds.) (1974), JefFrey \& KaWAhara (1982). We are pleased to use this occasion to mention the outstanding contribution of Professor J. ERICKSEN to the wave theory in nonlinear media (ERICKSEN, $1968,1971)$. Some aspects of wave fronts' behaviour in nonlinear dispersive media were considered also in the papers of Ivanov (1980) and BarenbLATT \& SHaPIRo (1984).

\section{Dispersive front model}

Hi

of

The analysis of the internal structure of wave fronts in dispersive media will be based here on a model equation:

$$
\partial_{t} u+u \partial_{x} u=M u+v \partial_{x x}^{2} u,
$$

in which $t$ is the time, $x$ is the coordinate normal to the front, $u$ is some characteristic of the motion which here for definiteness will be identified with the velocity, $v$ is the viscosity coefficient, $M$ is an operator governing dispersive and possibly some other effects within the front. Equation (1.1) can be obtained from more general equations of motion in a boundary-layer approximation. For this purpose the front thickness should be taken as a characteristic scale of length and after asymptotic analysis one should return in the simplified equation obtained to the original, dimension-bearing variables.

The results presented here seem to be applicable to a sufficiently wide class of operators $M$ although two specific operators only are considered in the present paper. The first of them is given by

$$
M u=-\beta \partial_{x x x}^{3} u .
$$

Here $\beta$, a constant, represents a certain dispersion coefficient. The equation obtained by this choice of operator is the well known Korteweg-de Vries-Burgers equation. SU \& GARDNER (1969) showed that this equation is fundamental in that it describes a wide class of Galilean-invariant, one-dimensional systems admitting motions with small nonlinearity, dispersion, and dissipation. It should be noted that this equation is not invariant with respect to the reflection $x^{\prime}=-x$. The second operator, namely

$$
M u=-2 \lambda^{2} \partial_{t x x}^{3} u-2 \lambda^{2} \partial_{x x}^{2}\left(u \partial_{x} u\right)
$$


where $\lambda$ is a constant having the dimensions of length, leads to an equation of the BENJAMIN, BoNA, \& MAHONY (1972) type, generalized by introducing nonlinear viscosity and dispersion. This equation, which was obtained by IVANOV (1980), is invariant like the Korteweg-de Vries-Burgers equation under Galilean transformations as well as reflections. Note that under certain conditions the equation corresponding to the operator (1.3) includes also negative viscosity effects and therefore can give rise to the instability and stochastic behavior.

Under external approximation when the spatial scale greatly exceeds the front width, the propagation of the front is described by a discontinuous solution of a complicated, and, in general, multidimensional system of equations. We shall restrict our attention here to the simplest case, that for which the equation of external approximation assumes the form

$$
\partial_{t} u+u \partial_{x} u=0
$$

so that in the external approximation the propagation of the front is described by a discontinuous solution of equation (1.4) of the progressive wave type:

$$
u=U(\xi) ; \quad U(\xi) \equiv u_{2} \text { when } \xi \leqq 0 ; \quad U(\xi) \equiv u_{1} \text { when } \xi>0 .
$$

Here $\xi=x-V t+c$ is the moving coordinate, $V=\left(u_{1}+u_{2}\right) / 2$ is the velocity of propagation of the front, and $c$ is a constant. The solution (1.5) of equation (1.4) settles at once into a step.

It is convenient in equation (1.2) to set $\beta=\lambda^{2} \Delta u, \Delta u=u_{2}-u_{1}$ and to consider simultaneously the Korteweg-de Vries-Burgers equation and the equation proposed in the paper by IVANOv (1980). If for equation (1.1) the initial condition $u(x, 0)$ is taken as a step, then the solution of the progressive wave type $u=U(\xi)$ will settle down, not at once but during a lapse of time of the order of $\tau_{1}=\lambda^{2} / \nu$.

The régime, i.e. the relative contributions of nonlinearity, dispersion and viscosity, is governed by a single dimensionless parameter, analogous to the Reynolds number, which is formed as follows from the wave amplitude $\Delta u=u_{2}-u_{1}$, the characteristic scale of length $\lambda$ and the viscosity $\nu$ :

$$
R=\lambda \Delta u / v \text {. }
$$

We stress additionally that the values $\lambda$ and $\Delta u$ can be considered as slowly varying functions of time, because equation (1.1) itself is obtained under a boundarylayer approximation.

Analysis of the steady wave solution $u=U(\xi)$ shows that for $R<R_{c}$ the velocity distribution has the form of a smoothed step (Figure 1, b). Moreover, if $R \ll 1$, the influence of the dispersion term is negligible small. Here $R_{c}$ is a certain critical value of the parameter $R$ of the order of unity. If $R>R_{c}$, the oscillations arise at the front: humps of wave length $\sim \lambda$ are generated. The thickness $\Lambda$ of the front (the width of the region in which the velocity $u$ varies greatly) is of the order $\Lambda \sim \lambda R$. If $R \gg 1$, many oscillations arise at the front. The time of separation of one hump has the order of magnitude $\tau_{0}=\lambda / \Delta u$, and so we obtain $\tau_{1}=R \tau_{0} \gg \tau_{0}$ : the gross time for the front to settle is much greater for fronts with large $R$ than the time of separation of one hump. 
When $R \gg 1$, the fronts having many oscillations for which the contribution of dispersion is high we shall call henceforth dispersion fronts. The present paper is devoted basically to analysis of the structure of dispersion fronts.

\section{Smoothing. The equations for properties of the averaged motion}

For the dispersion fronts it is worthwhile to consider the space-averaged properties of the motion for instance, the space-averaged velocity

$$
\vec{u}(x, t)=\frac{1}{2 L} \int_{x-L}^{x+L} u(\xi, t) d \xi
$$

The averaging length scale $L$ has to be taken much larger than the length of a single hump, but, at the same time, much less than the front thickness $A$. Under such conditions space averaging and differentiation with respect to $x$ and $t$ commute.

Averaging equation (1.1), we reduce it to the form

$$
\partial_{t} \bar{u}+\bar{u} \partial_{x} \bar{u}=M \bar{u}+\partial_{x}\left[\left(v+v_{e}\right) \partial_{x} \bar{u}\right]
$$

Here the effective viscosity $v_{e}$ is defined as follows:

$$
v_{e}=-\frac{b}{\partial_{x} \bar{u}}, \quad v_{e}=-\frac{b+2 \lambda^{2} \partial_{x x}^{2} b}{\partial_{x} \bar{u}} ; \quad b=\frac{\overline{u^{\prime 2}}}{2}
$$

for the operators (1.2) and (1.3), respectively and a new notation for the velocity "fluctuation" $u^{\prime}=u-\bar{u}$ is introduced. The appearance of the effective velocity $v_{e}$ is due to momentum transfer by the humps going out of the front. The order of magnitude of the effective viscosity is given by $v_{e} \sim \Delta u \Lambda$, and so the ratio $v_{e} / \boldsymbol{v}$ is very large for dispersion fronts because $v_{e} / \nu \sim[(\Delta u \lambda) / \nu] A / \lambda=$ $R A / \lambda \gg R \gg 1$. We can therefore neglect $\nu$ in equation (2.2) in comparison with $v_{e}$. Thus the averaged equation has the same form as the original one, but the effective viscosity $v_{e}$ appears in place of the actual one.

Let us introduce the effective Reynolds parameter corresponding to the averaged equation (2.2):

$$
R_{e f}=\lambda \Delta u / v_{e} .
$$

We obtain $R_{e f}=R v / v_{e} \sim \lambda / \Lambda \ll 1$, and so we can neglect also the term with the operator $M \bar{u}$ in the averaged equation (2.2). Thus an equation of the Burgers type with variable viscosity appears for the averaged velocity:

$$
\partial_{t} \bar{u}+\bar{u} \partial_{x} \bar{u}=\partial_{x}\left(\nu_{e} \partial_{x} \bar{u}\right)
$$

We note furthermore that the term $2 \lambda^{2} \partial_{x x}^{2} b$ in the second expression for the effective viscosity (2.3) is small in comparison with the first one, $b$. In fact, we have $\lambda^{2} \partial_{x x}^{2} b / b \sim \lambda^{2} / \Lambda^{2} \ll 1$. We can therefore in both cases define the effective viscosity in the dispersion front in the same way:

$$
v_{e}=-b / \partial_{x} \vec{u}
$$




\section{The limiting régimes of the dispersion-front evolution}

As we noted previously, the asymptotic form of the progressive wave type settles down at the front during a period of the order $\tau_{1}=\lambda^{2} / \nu$. Substituting into equation (2.5) $\bar{u}=\vec{u}(\xi), \quad \xi=x-V t+c, \quad V=\frac{1}{2}\left(u_{1}+u_{2}\right), \quad c=$ const, $\nu=v_{e}(\xi)$ and integrating the simple equation obtained, we derive the relation between $v_{e}$ and $\bar{u}$ :

$$
v_{e}=-\frac{1}{2}\left(u_{2}-\bar{u}\right)\left(\bar{u}-u_{1}\right) \partial_{\xi} \bar{u} .
$$

Comparing (3.1) with (2.6), we obtain for the limiting steady régime a relation between averaged velocity and "fluctuation energy" - half of the mean square of velocity fluctuation-

$$
b=\frac{1}{2} \overline{u^{\prime 2}}=\frac{1}{2}\left(u_{2}-\bar{u}\right)\left(\bar{u}-u_{1}\right) .
$$

The intermediate-asymptotic régime is also of interest for dispersion fronts, when the times are much larger than the time $\tau_{0}$ for a single hump to separate and also are much less than the time for the motion to settle into the steady régime $\tau_{1}$ :

$$
\tau_{1}>t \gg \tau_{0}
$$

This intermediate-asymptotic regime corresponds to a self-similar solution of the equation (2.5) governed by following parameters: $x-u_{1} t, \Delta u, t$. By dimensional analysis we obtain

$$
\bar{u}=u_{1}+\Delta u U(\zeta), \quad \zeta=\frac{x-u_{1} t}{t \Delta u}, \quad v_{e}=(\Delta u)^{2} t G(\zeta)
$$

Here $U(\zeta)$ and $G(\zeta)$ are the dimensionless functions connected by a relation obtained after substituting (3.4) into (2.5) and integrating:

$$
G(\zeta)=-\left[\left(1-U^{2}\right)-2 \zeta(1-U)+2 \int_{-\infty}^{\zeta}(1-U) d \zeta\right] /(2 d U / d \zeta)
$$

From the last equation and (2.6) we obtain

$$
b=\frac{1}{2}(\Delta u)^{2}\left[1-U^{2}-2 \zeta(1-U)+2 \int_{-\infty}^{\zeta}(1-U) d \zeta\right] .
$$

\section{Asymptotic and numerical analysis of the effective viscosity}

We obtained in the preceding Section a relation between effective viscosity, fluctuation energy and averaged velocity gradient. We started from the assumption that certain limiting régimes settle down at the front: the self-similar régime in the time range $\tau_{1}>t \gg \tau_{0}$ and the progressive wave régime for $t \gg \tau_{1}$.

For the purpose of confirming these assumptions more detailed asymptotic analysis of the effective viscosity could be of interest as would also comparison of the results of such analysis with numerical calculations.

We note first of all that when $t \ll \tau_{1}$ we can neglect the effects of actual viscosity. Therefore the Korteweg-de Vries equation is obtained for the first 
of the models under consideration. An asymptotic analysis of the stepwise initial distribution evolution was performed by GuREvich \& PITAEvsky (1973). According to their results

$$
u(x, t)=u_{1}+\Delta u\left\{2 \mathrm{dn}^{2}[(x-V t) / \lambda \sqrt{6}, m]-1+m\right\}
$$

where the velocity of propagation of the wave is

$$
V=u_{1}+(1+m) \Delta u / 3
$$

and the amplitude of the oscillation is

$$
u_{\max }-u_{\min }=2 m \Delta u .
$$

Here dn $(z, m)$ is the Jacobian elliptic function (Abramovitz \& Stegun (1964)). The parameter $m$ is related to $x$ and $t$ by a self-similar relation

$$
\zeta=\frac{x-u_{1} t}{t \Delta u}=\frac{1+m}{3}-\frac{2}{3} \frac{m(1-m)}{\varphi(m)-1+m},
$$

where the notation $\varphi(m)=E(m) / K(m)$ is used, $E(m)$ and $K(m)$ being the complete elliptic integrals of modulus $k=\sqrt{m}$. The parameter $m$ varies slowly from the head of the front, where $m=1, \zeta=2 / 3$, to the trail of the front, where $m=0$, $\zeta=-1$. Using the properties of the elliptic functions, we obtain for the hump length

$$
l=2 \sqrt{6} \lambda K(m),
$$

so and in accordance with the estimates of Section 1 this quantity is of the order of $\lambda$ and diminishes slowly from head to tail.

The relation (4.1) allows one to find the velocity averaged over the period of oscillation:

$$
\bar{u}=u_{1}+\Delta u(2 \varphi(m)-1+m) .
$$

Estimating with the help of (4.1) also the quantities $\partial_{x} \bar{u}$ and $b=\frac{1}{2} \overline{(u-\bar{u})^{2}}$, we obtain

$$
\begin{aligned}
\partial_{x} \bar{u} & =\frac{1}{t m} \frac{3\left[2 \varphi-1+m-\varphi^{2} /(1-m)\right](\varphi-1+m)^{2}}{(1-m)(2-3 m)-2 \varphi(1-2 m)}, \\
b & =(\Delta u)^{2}\left[-2 \varphi^{2}+(4 / 3) \varphi(2-m)+(2 / 3)(m-1)\right] .
\end{aligned}
$$

From these formulae a parametric expression could be obtained for the dimensionless effective viscosity $G(\zeta)=v_{e} /(\Delta u)^{2} t$ at the intermediate asymptotic stage (3.3) of the evolution of the front:

$$
G(\zeta)=-\frac{\left[\frac{4}{3} \varphi(2-m)-2 \varphi^{2}-\frac{2}{3}(1-m)\right] m[(1-m)(2-3 m)-2 \varphi(1-2 m)]}{3\left[2 \varphi-1+m-\phi^{2} /(1-m)\right](\varphi-1+m)^{2}} .
$$

Here the relation between $m$ and $\zeta$ is given by the equation (4.4). The graph of the function $G(\zeta)$ is given in Figure 2a. We seethat within the basic part of the front the function $G(\zeta)$ is nearly constant. 
initial

cord-

64)).

plete

the

$=0$, ump

order

d of
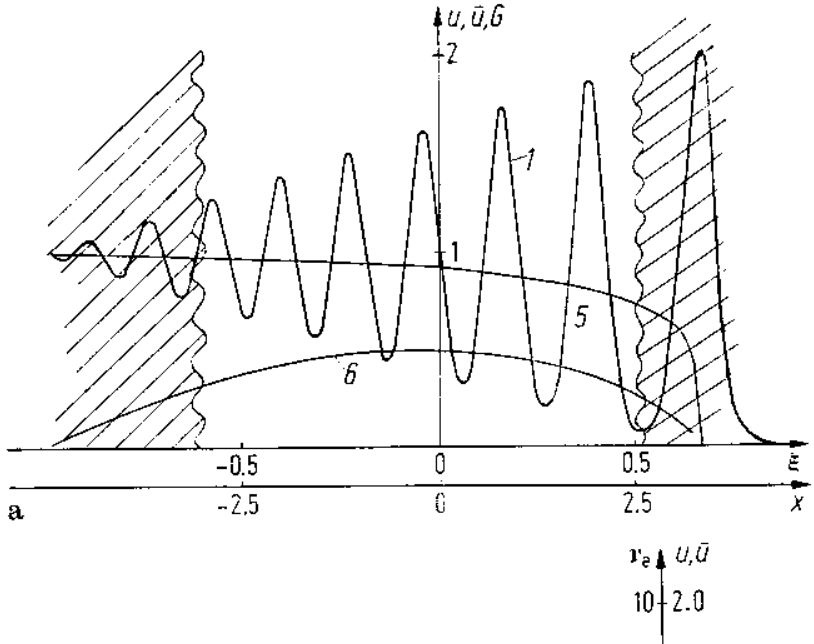

b
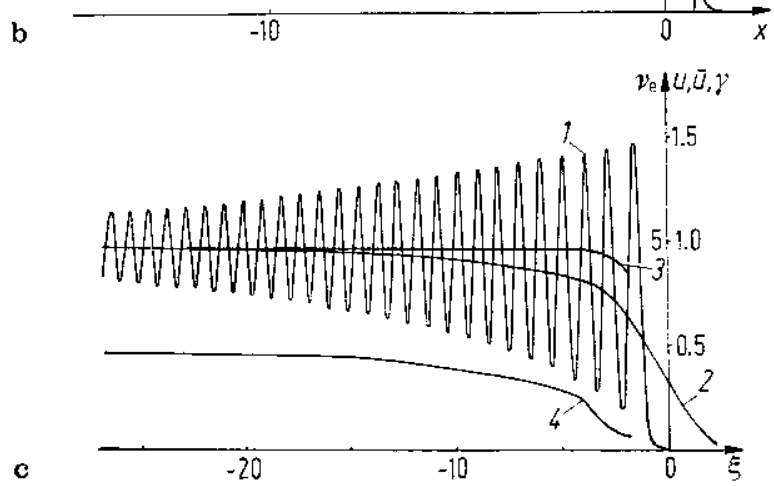

Fig. 2. The numerical calculation of the stepwise initial distribution evolution (operator (1.2)): $u(x, 0) \equiv 1$ at $x \leqq 0, u(x, 0) \equiv 0$ at $x>0 ; v=10^{-3}, \lambda=10^{-1}, R=100$. (a) $t=5<\tau_{1}=\lambda^{2} / v$. (b) $t=10 \sim \tau_{1}$. (c) $t=\infty$. The results show that in the basic part of the front the effective viscosity $v_{e}$ is nearly constant and for $t \geq \tau_{1}$ is of the order of unity. This gives in accordance with (4.16) $v_{e} / v \sim 10^{4}$. (1) the actual velocity distribution $u(x, t)$. (2) the averaged velocity distribution $\bar{u}(x, t)$, (3) the ratio $\gamma$ of effective viscosity to its approximate value, calculated by formula (3.1), (4) the effective viscosity $v_{e}$ calculated according its definition by the formula $(2.3),(5) \bar{u}(\zeta)$, see formula (4.6),

(6) $G(\zeta)$, see formula (4.9) 
At times of the order of $\tau_{1}=\lambda^{2} / \nu$ the influence of actual viscosity starts to be essential. Therefore the solution of the equation (1.1) for the initial conditions of the step type and both operators (1.2) and (1.3) was calculated numerically. The calculations employed an implicit divergent-difference scheme giving the second-order approximation in time and fourth-order approximation in space. This scheme is described in detail in the paper by ZaKHAROV, Ivanov \& KoRETSKY (1981). The values of $u(x, t), v_{e}(x, t), \vec{u}(x, t)$ computed when the operator (1.2) was used and $u_{2}=1, u_{1}=0, \lambda=10^{-1}, v=10^{-3}$ are presented in Figure $2 b$ for $t=10$. When the operator (1.3) was used and $u_{2}=1, u_{1}=-1$ the computed values of the same quantities are presented in Figure $3 \mathrm{a}$ for $t=5$. The results of numerical analysis show that the quantity $v_{e}$ is of the order of unity which confirms the assumption made above that $v_{e} \gg v$.

When $t \gg \tau_{1}$ the dispersion front becomes steady. The equation of steady front admits an asymptotic solution, allowing the effective viscosity to be calculated directly.
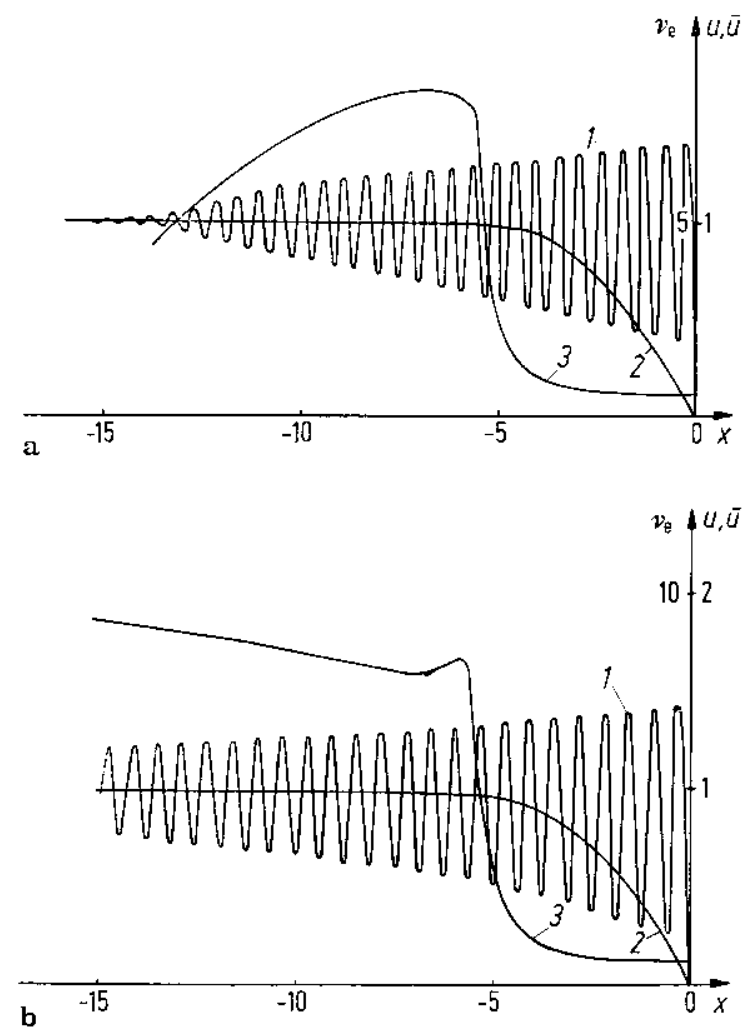

Fig. 3. The numerical calculation of the stepwise distribution evolution (operator (1.3)): $u(x, 0)=1$ at $x \leqq 0, u(x, 0)=-1$ at $x>0 ; v=10^{-3}, \lambda=\frac{10^{-1}}{\sqrt{2}}, \quad R=100 \sqrt{2}$ (a) $t=5$. (b) $t=\infty$. The numbers of the curves correspond to the same quantities as for the Figure 2 
Substituting $u=U(\xi)$ into equation (1.1), using the boundary condition and introducing dimensionless variables, by integration we obtain for the operator (1.2)

where

$$
\frac{d^{2} W}{d \eta^{2}}+\frac{W}{2}=-\varepsilon\left(-\frac{d W}{d \eta}+\frac{W^{2}}{2}\right)
$$

$$
\varepsilon W=\left(u-u_{1}\right) / \Delta u-1, \quad \eta=\xi / \lambda, \quad \varepsilon=1 / R .
$$

The parameter $\varepsilon$ is small for dispersion fronts, this very smallness allows us to use an asymptotic method (BoGolubov \& MiTROPOLSKY (1974)). We search for the solution of the equation (4.10) in the form

$$
W=a \cos \psi+\varepsilon W_{1}(a, \psi)+O\left(\varepsilon^{2}\right) .
$$

Here $a$ and $\psi$ are new unknown functions. Effecting asymptotic integration of the equations obtained for these functions, we obtain

$$
W_{1}=-\frac{1}{2} a^{2}\left(1-\frac{1}{3} \cos 2 \psi\right),
$$

where the variable amplitude $a$ and phase $\psi$ of oscillation satisfy the equations

$$
\frac{d a}{d \eta}=\varepsilon \frac{a}{2}+O\left(\varepsilon^{2}\right), \quad \frac{d \psi}{d \eta}=\frac{1}{\sqrt{2}}+O\left(\varepsilon^{2}\right)
$$

For the quantities $\bar{u}, \partial_{\xi} \bar{u}$ and $b$ averaged over the phase of oscillation $\psi$ the expressions

$$
\bar{u}=u_{2}-\Delta u \frac{\varepsilon^{2} a^{2}}{2}, \quad \partial_{\xi} \bar{u}=-\Delta u \frac{\varepsilon^{3} a^{2}}{2 \lambda}, \quad b=\frac{(\Delta u)^{2} \varepsilon^{2} a^{2}}{4}
$$

are obtained, and so the effective viscosity $v_{e}$ turns out to be constant:

$$
v_{e}=\frac{1}{2} \nu R^{2} \text {. }
$$

The functions $u(\xi), \bar{u}(\xi), b(\xi)$ and $v_{e}(\xi)$ were obtained also by numerical integration of equation (4.10). The results of that integration when $\varepsilon=10^{-2}$ are presented in Figure $2 \mathrm{c}$. The averaging was performed by low-frequency filtration of the solution with the Hanning weighting cosinus-filtre (BENDAT \& PIERSOL, 1966). The calculations confirmed the conclusion that the value of the effective viscosity $v_{e}$ is several orders higher than the actual viscosity.

The asymptotic analysis of the progressive wave front for the operator (1.3) is performed analogously. The following asymptotic representation for the velocity is obtained:

$$
u=u_{1}+\Delta u\left[1+\varepsilon a \cos \psi-\frac{1}{2} \varepsilon^{2} a^{2}(1+\cos 2 \psi)+O\left(\varepsilon^{3}\right)\right],
$$

the amplitude $a$ and phase $\psi$ being governed by the equations

$$
\frac{d a}{d \xi}=\frac{\varepsilon a}{2 \lambda}+O\left(\varepsilon^{2}\right), \quad \frac{d \psi}{d \xi}=\frac{1}{\sqrt{2} \lambda}+O\left(\varepsilon^{2}\right) .
$$


Further analysis leads again to the expression (4.16) for the effective visocisty. This coincidence seems not to be accidental; it demonstrates the universality of the effective viscosity mechanism in the dispersion front.

Thus numerical calculations and asymptotic analysis lead to consistent results confirming the basic dispersion-front model presented here.

\section{Closure of the equation for the averaged field}

It is shown in the preceding sections that the averaged velocity field in the dispersion front is governed by the Burgers equation with variable effective viscosity $\nu_{e}$ :

$$
\partial_{t} \bar{u}+\bar{u} \partial_{x} \bar{u}=\partial_{x}\left(\nu_{e} \partial_{x} \bar{u}\right)
$$

where the effective viscosity is related to fluctuation energy $b=\sqrt{\frac{1}{u^{\prime 2}}}$ and the averaged velocity gradient by the equation

$$
b=-v_{e} \partial_{x} \bar{u} \text {. }
$$

The analogy with turbulence theory is clear here: equation (5.1) is not closed because $\nu_{e}$ is unknown; the formula (5.2) is completely analogous to the Prandt formula, well known in turbulence theory. The analysis presented above allows one to propose simple closure schemes for the equation (5.1) analogous to semiempirical turbulence theories. As in those theories, closure is obtained by assuming an expression relating effective viscosity with average velocity derivatives. Such an assumption closes equation (5.1); by solving it, we can find the averaged field $\vec{u}(x, t)$. That allows us to find in turn, by use of equation (5.2), the mean-square velocity fluctuation:

$$
\left[\overline{u^{\prime 2}}\right]^{\frac{1}{2}}=\left[2 v_{e}\left|\partial_{x} \bar{u}\right|\right]^{\frac{1}{2}}
$$

What seems to be the simplest assumption of closure is

$$
v_{e}=\alpha \Lambda \Delta u \text {. }
$$

Here $A$ is the thickness of the front and $\alpha$ is a constant. Let us consider what application of the formula (5.4) gives for limiting régimes. At the initial stage of front propagation when $t$ is still less than the settling time $\tau_{1}$ for the steady régime although much larger than the time of separation of a single hump, the actual viscosity effect can be neglected, and so a Gurevich-Pitaevskey self-similar régime sets in (see Section 4). For this régime $A=t \Delta u$.

The simplest condition to determine the constant $\alpha$ seems to follow if we require that at $t=\tau_{1}=\lambda^{2} / v$ the value of $v_{e}$, determined by the equation (5.4), should coincide with the value $v_{e}=\frac{1}{2} \nu R^{2}$ obtained in the Section 4 for the steady régime. We obtain $\alpha(\Delta u)^{2} t_{1}=\alpha(\Delta u)^{2} \lambda^{2} / \nu=\frac{1}{2} v R^{2}$, whence $\alpha=0,5$. As Figure 2a shows, the value of $\alpha$ is in fact nearly constant outside the first few humps, i.e. in the basic region of the front. When $t>\tau_{1}$ the front width could be taken as the constant $\Lambda=R \lambda$, and so an expression $v_{e}=\frac{1}{2} v R^{2}$ can be taken for the effective viscosity. Note that equation (1.1) describes the front structure in a boundary-layer approximation, and so the quantities $\lambda$ and $\Delta u$ entering the expression for $R$ could be slowly varying functions of time. Compari- 
son of the effective viscosity obtained for a steady front according to the equation (3.1) and by numerical integration is presented in Figure 2c. As we see, the approximation $v_{e}=\frac{1}{2} \nu R^{2}$ is rather satisfactory if the first few humps are excluded. Calculation of the fluctuation energy $b$ by the relation (5.2) and the expression adopted for the effective viscosity also gives satisfactory results: the error in the mean-square velocity fluctuation does not exceed $50 \%$, and the difference between averaged velocity, found from solving the equation (5.1) and the exact one, does not exceed $15 \%$. For the applications we have in mind such an approximation is satisfactory. We note, however, that more accurate closure schemes for the averaged field equation of the dispersion front have been proposed (BARENBLATT \& SHAPIRO (1984)), corresponding to advanced approaches of semi-empirical turbulence theory.

\section{References}

Abramowitz, M., \& I. A. Stegun. 1964. Handbook of mathematical functions, NBS, Applied Mathematics series 55.

Barenblatt, G. I., \& G. I. Shapiro. 1984. Bull. USSR Ac. of Sci. Ser. Atm. and Ocean Physics 19, 277-284.

Bendat, J. S., \& A. G. Piersol. 1966. Measurement and analysis of random data. John Wiley \& Sons, Inc. N.Y., London, Sydney.

Benjamin, T. B., J. L. Bona, \& J. J. Mahony. 1972. Phil. Trans. Roy. Soc. London A 272, 47-78.

Bogolubov, N. N., \& YU. A. Mitropolsky. 1974. Asymptotic methods in nonlinear oscillations theory. Nauka, Moscow (in Russian).

ERICKSEN, J. L. 1968. Journal of the Acoustical Society of America 44, 444-446.

ERICKSEn, J. L. 1971. Archive for Rational Mechanics and Analysis 43, 167-178.

Gaponov, A. V., L. A. Ostrovsky, \& G. I. Freidman. 1967. Radiophysics, 10, 13761413.

Gurevich, A. V., \& L. P. Pitaevsky. 1973. Sov. Phys. JETP. 65, 590-604.

IvaNOV, M. YA. 1980. Numerical methods of continuum mechanics 11, 75-90.

Jefreey, A., \& T. Kawahara. 1982. Asymptotic methods in non-linear wave theory, Pitman, Boston, London, Melbourne.

KARPMAN, V. I. 1973. Non-linear waves in dispersive media, Nauka, Moscow (in Russian).

Kochin, N. E. 1926. Rendiconti del Circolo Matematico di Palermo 50, 305-344.

Leibovich, A., \& A. R. Seebass (ed.). 1974. Non-linear waves. Cornell University Press, Ithaca and London.

MonIN, A. S., \& A. M. YAGLom. 1971. Statistical fluid mechanics: Mechanics of Turbulence, vol. 1, MIT Press, Cambridge and London.

Sagdeev, R. Z. 1964. In Topics of plasma theory, M. A. Leontovich \& L. A. ARtsimoVICH (eds.), vol. 5, Atomizdat, Moscow (in Russian).

Su, C. H., \& G. S. Gardner. 1969. J. Math. Phys. 10, 536-539.

Whтнам, G. 1974. Linear and non-linear waves. J. Wiley \& Sons, N.Y., London, Sydney, Toronto.

Zakharov, V. D., M. Ya. Ivanov \& V. V. Koretsky. 1981. In Problems of viscous flows. Novosibirsk (in Russian), 65-71.

P.P. Shirshov Institute of Oceanology, USSR Acad. of Sci, 23, ul. Krasikova, Moscow 117218, USSR

(Received April 11, 1984) 